\title{
Research on Hot-Wire Low Pressure Wind Speed Measurement System
}

\author{
Yin Xiaofang ${ }^{1, a}$, Li Xiyuan ${ }^{1}$, Gao Qinghua ${ }^{1}$ and Wang Jing ${ }^{1}$ \\ ${ }^{1}$ Beijing Institute of Spacecraft Environment Engineering, Beijing 100094, China
}

\begin{abstract}
In order to verify the correctness of the thermal control design, the Mars rover needs to conduct a thermal test on the ground during the construction of the Mars exploration mission. In the course of the thermal test, real-time measurement of the wind speed at low pressure is required. Therefore, it is needed to establish a low-pressure wind speed measurement system. This article describes the composition of the test system, the choice of measurement sensors, in which the impact of wire resistance, electromagnetic interference and other factors are taken into account, and the design of the test process is completed.
\end{abstract}

\section{Introduction}

Mars also enveloped the atmosphere, similar to the Earth's atmosphere. Mars's main component of the atmosphere is carbon dioxide, followed by nitrogen, argon, in addition to a small amount of oxygen and water vapor. There is nitrogen in both the Martian atmosphere and the Earth's atmosphere, which is the biggest similarity between Mars and the Earth. The density of the atmosphere in Mars is less than one percent of the Earth's atmosphere, the surface atmospheric pressure 500-700pa[1-2].

According to previous tests, the convective heat transfer coefficient at $1000 \mathrm{~Pa}$ low pressure can be measured by the wind speed sensor even though the relative atmospheric pressure is reduced. After re-calibration of the hot-wire wind speed sensor, the convection heat transfer coefficient can be used for low pressure[3].

\section{Hot-wire low pressure wind speed measurement system[4-5]}

\subsection{Hot-wire anemometer measurement principle}

Hot air anemometer measurement circuit diagram is as follows:

\footnotetext{
a Corresponding author : 190064602@qq.com
} 


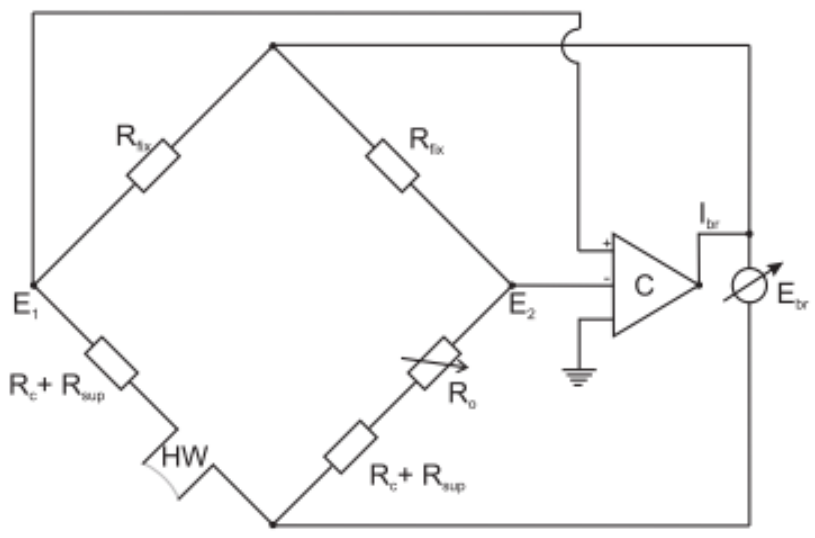

Figure 1 bridge measurement circuit

As shown in the figure 1, where Rfix is the internal resistance of the bridge, Rc + Rsup is the resistance of the wire + the resistance of the bracket. In order to accurately measure the hot wire HW, it is necessary to ensure that $\mathrm{Rc}+$ Rsup does not change during the test. Can not change too much. The system limits are as follows:

a) The cable resistance changes little during the test. The wire resistance and calibration state are the same when measuring.

b) The system needs a brush slip ring;

c) Since the wind speed sensor is a high-frequency analog signal, electromagnetic interference needs to be considered.

Aiming at these constraints, the system diagram of wind speed measurement under low pressure is as shown in Figure 2:

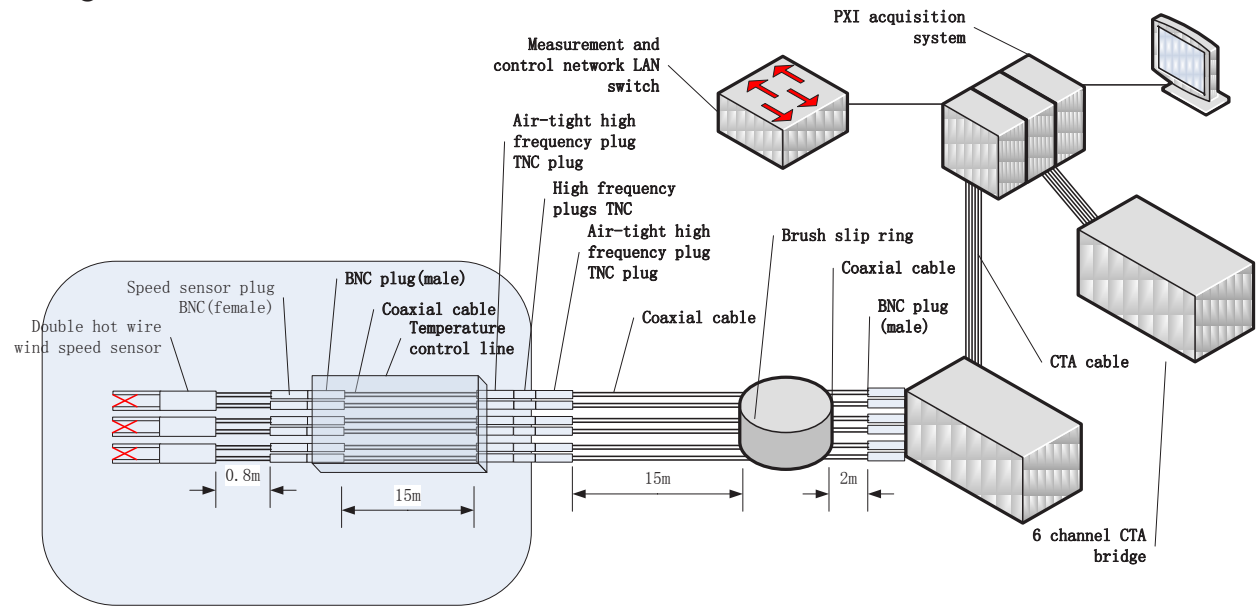

Figure 2 low pressure wind speed measurement system

The following figure shows the wind speed measurement system in calibration mode:

As can be seen from the figure 3, the system includes two parts of the cable inside the container and the cable outside the container, wherein the cable inside the container is a slip ring in the calibration mode, and the $15 \mathrm{~m}$ coaxial cable is outside the container. Wind speed measurement mode, the exchange of the cable inside and outside the container, to ensure that the calibration and use of the state of the same resistance. At the same time, in the calibration and use mode, all through the aluminum temperature control temperature of the cable temperature, reduce the temperature changes on the cable resistance. 


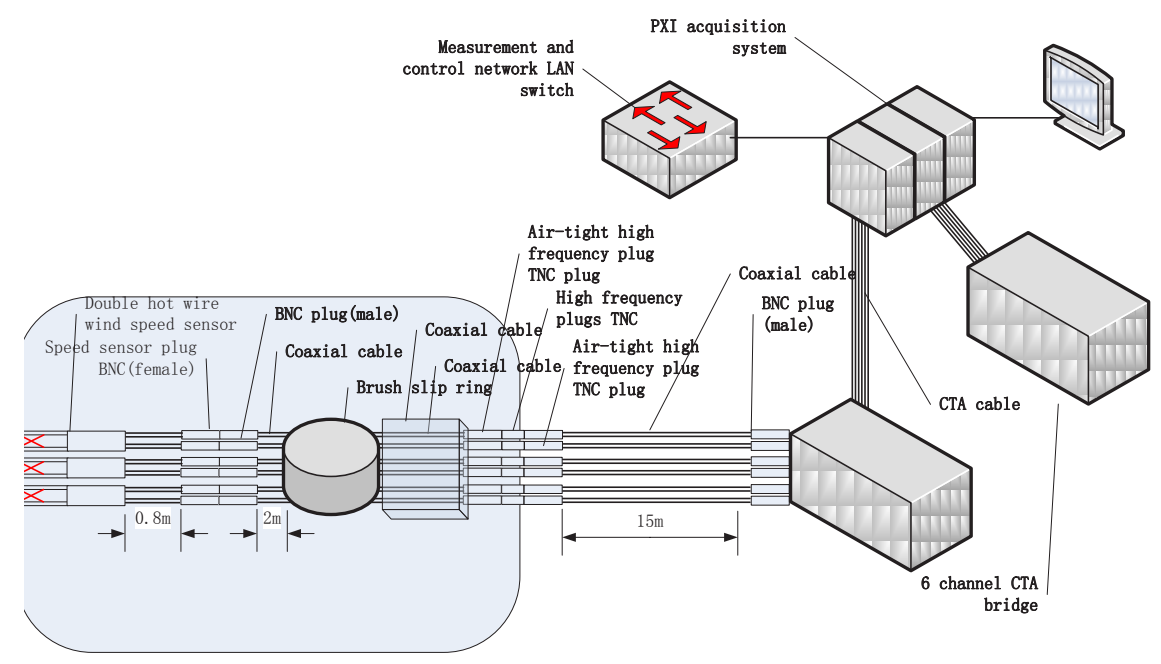

Figure 3 low pressure wind speed measurement system (calibration mode)

\subsection{System Configuration}

According to preliminary tests, the use of 55R01 series coated hot wire sensor can be under low pressure on the wind speed measurement accuracy, select the same type of $\mathrm{x}$-type probe. In order to meet the wind speed measurement requirements of different directions, select two types of wind speed probe, model 55R52 and 55R51 respectively, as shown in the Figure 4:
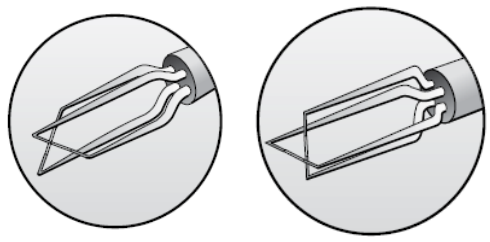

Figure 4 55R51 and 55R52 probe

One probe are 0.5 um coating, each resistance is about $6 \Omega$, the temperature coefficient of $0.44 \%$ $/{ }^{\circ} \mathrm{C}$, the maximum temperature of $300{ }^{\circ} \mathrm{C}$. According to the test at low pressure, the sensitivity of the probe is about $4-8 \mathrm{mv} /(\mathrm{m} / \mathrm{s})$, as shown in Figure 5:

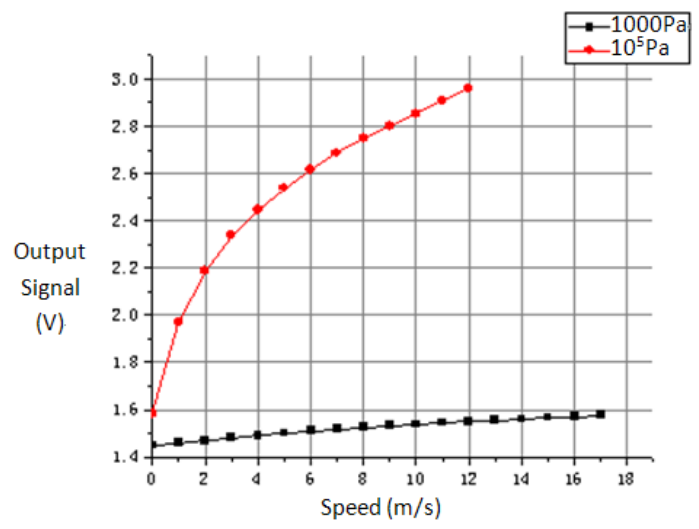

Figure 5 probe output at low pressure

Measurement accuracy to meet the requirements of the wind speed can be accurately measured. 
Probe support played a role in the wind speed probe support and signal transmission, in order to take into account the various test point of view, select the two probe holder, respectively, straight probe and $90^{\circ}$ probe, as shown in Figure 6 and Figure 7:

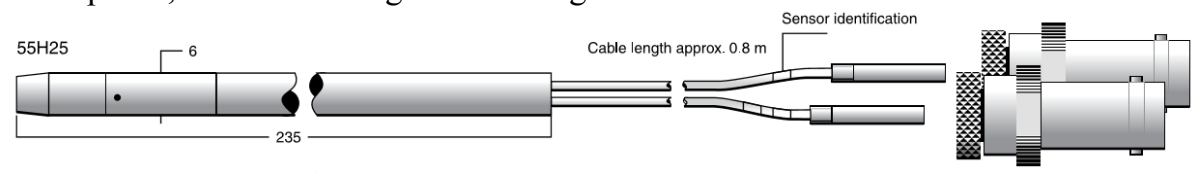

Figure 6 9055H025 Probe Holder

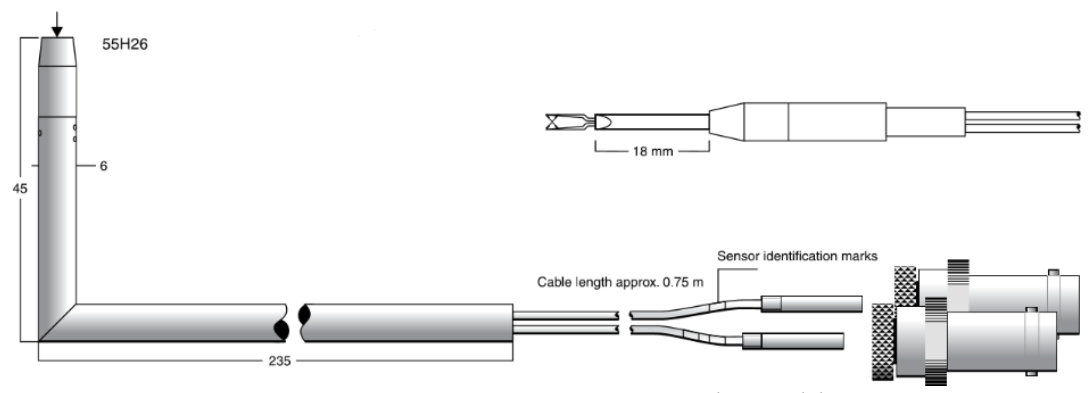

Figure 7 9055H026 Probe Holder

Each two-dimensional wind speed probe occupies two bridges of the bridge and a total of 12 probes are required for the six probes. Two Dantec 6-channel bridges with six wind speed measurement channels and one platinum resistance temperature measurement channel are available Consider the influence of temperature on wind speed measurement. Dantec 6-channel wind speed measurement system is shown in Figure 8.

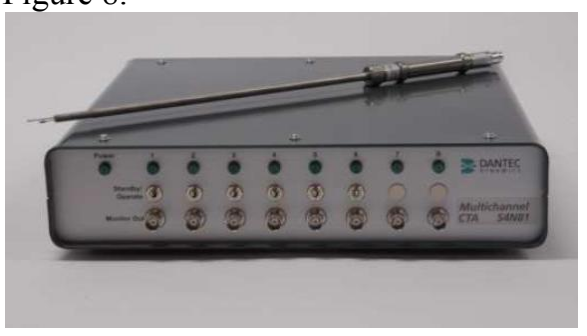

Figure 8 Dantec 6-channel wind speed measurement system

The data acquisition system chooses NI Corporation based on the PXI measuring instrument, The program can measure the $0-5 \mathrm{~V}$ signal output by the wind speed probe, of which 24-bit accuracy of up to $1.192 \mathrm{uV}$, to meet the measurement requirements.

There are two types of measurement cables: Slip-free and Slip-ring, respectively defined as A and B cables, A cable used to measure the status of the in-container cable and the calibrated out-of-container cable, B cable used to measure the status of Container outside the cable and calibration of the container cable, A, B each requires two group.

The use of constant heat flow to drive the wind speed probe, the probe temperature is constant temperature, the total heat loss through the probe:

$$
Q=s h \Delta t=\frac{s N u \lambda}{1} t_{w}-t_{e}
$$

Which $\mathrm{Nu}$ can be expressed as a function of Re, due to the cylinder, sphere approximation, and the ball has been under low pressure for many times to verify the ball, for example, can be expressed as:

$$
N u=2+\left(0.4 R e^{0.5}+0.06 R e^{2 / 3}\right) R r^{0.4}
$$

According to the test at low pressure, the output of the wind speed sensor calculated by this formula at $40000 \mathrm{~Pa}$ is completely consistent with the atmospheric pressure, but the deviation is about 
$20 \%$ at about $1000 \mathrm{~Pa}$. At present, according to the literature, the $\mathrm{Nu}-\mathrm{Re}$ number caused by low atmospheric pressure is usually correlated According to the test, at about $700 \mathrm{~Pa}$ and $0-15 \mathrm{~m} / \mathrm{s}$, the equation can be modified by the formula $: \mathrm{h} *(\mathrm{P} 1 / \mathrm{P} 0) 2$

$$
h=h_{0} \times\left(\frac{P_{1}}{P_{0}}\right)^{0.07}
$$

Where $\mathrm{P} 1$ and $\mathrm{P} 2$ are the operating pressure and atmospheric pressure, respectively, and h0 is the convective heat transfer coefficient calculated according to the criterion. The corrected $\mathrm{h}$ can be used to evaluate the output of the wind speed sensor under low pressure.

The picture of turntable calibration system is in Figure 9:
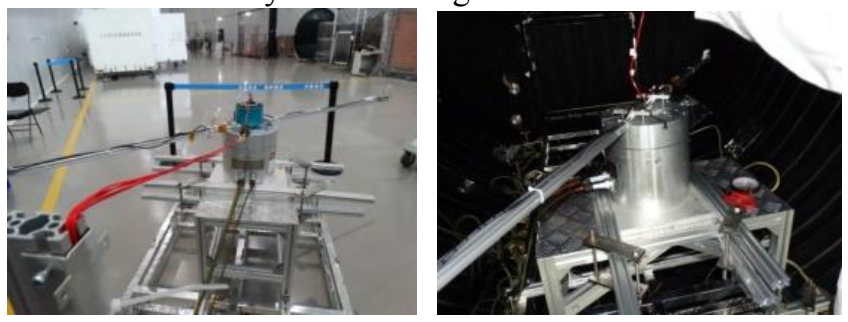

Figure9 turntable calibration system

\subsection{Systematic Error Analysis}

According to the previous simulation of the flow field near the cantilever using the CFD simulation method, the influence of the cantilever itself on the flow field can be reduced beyond $0.1 \mathrm{~m}$ outside the cantilever, reducing the error level to about $0.2 \mathrm{~m} / \mathrm{s}$.

Turntable speed can be adjusted between $0 \sim 100 \mathrm{RPM}$, the diameter of the cantilever $2.6 \mathrm{~m}$, according to the formula :

$$
V=R P M \cdot \frac{\pi R}{60}=\frac{\pi}{60} \cdot R P M \cdot R
$$

The error for speed is

$$
d V=\frac{\pi}{60} \cdot[d(R P M) \cdot R+R P M \cdot d(R)]
$$

The RPM error is less than 0.5RPM. The error of radius $\mathrm{R}$ is measured with ruler and the error is less than $0.5 \mathrm{~mm}$. The error of wind speed calibration can be calculated for each working condition. In actual calibration, the combination of pressure, temperature and output needs to be calibrated with the wind speed. Under each combination of pressure and temperature, the calibrated wind speed condition

\begin{tabular}{|c|c|c|c|c|c|c|c|}
\hline No. & $\begin{array}{l}\text { Wind speed } \\
\mathrm{m} / \mathrm{s}\end{array}$ & $\begin{array}{l}\text { Rotating } \\
\text { speed(RPM) }\end{array}$ & error & No. & $\begin{array}{l}\text { Wind speed } \\
\mathrm{m} / \mathrm{s}\end{array}$ & $\begin{array}{l}\text { Rotating } \\
\text { speed(RPM) }\end{array}$ & error \\
\hline 1 & 0.5 & 3.67 & 0.03 & 21 & 10.5 & 77.13 & 0.04 \\
\hline 2 & 1 & 7.35 & 0.03 & 22 & 11 & 80.8 & 0.04 \\
\hline 3 & 1.5 & 11.02 & 0.03 & 23 & 11.5 & 84.47 & 0.04 \\
\hline 4 & 2 & 14.69 & 0.03 & 24 & 12 & 88.15 & 0.04 \\
\hline 5 & 2.5 & 18.36 & 0.03 & 25 & 12.5 & 91.82 & 0.04 \\
\hline 6 & 3 & 22.04 & 0.03 & 26 & 13 & 95.49 & 0.04 \\
\hline
\end{tabular}
is initially determined as shown in Table 1 :

Table 1 calibration conditions 


\begin{tabular}{|r|r|r|r|r|r|r|r|}
\hline 7 & 3.5 & 25.71 & 0.03 & 27 & 13.5 & 99.17 & 0.04 \\
\hline 8 & 4 & 29.38 & 0.03 & 28 & 14 & 102.84 & 0.04 \\
\hline 9 & 4.5 & 33.06 & 0.03 & 29 & 14.5 & 106.51 & 0.04 \\
\hline 10 & 5 & 36.73 & 0.03 & 30 & 15 & 110.18 & 0.04 \\
\hline 11 & 5.5 & 40.4 & 0.04 & 31 & 15.5 & 113.86 & 0.04 \\
\hline 12 & 6 & 44.07 & 0.04 & 32 & 16 & 117.53 & 0.04 \\
\hline 13 & 6.5 & 47.75 & 0.04 & 33 & 16.5 & 121.2 & 0.04 \\
\hline 14 & 7 & 51.42 & 0.04 & 34 & 17 & 124.88 & 0.04 \\
\hline 15 & 7.5 & 55.09 & 0.04 & 35 & 17.5 & 128.55 & 0.04 \\
\hline 16 & 8 & 58.76 & 0.04 & 36 & 18 & 132.22 & 0.04 \\
\hline 17 & 8.5 & 62.44 & 0.04 & 37 & 18.5 & 135.89 & 0.04 \\
\hline 18 & 9 & 66.11 & 0.04 & 38 & 19 & 139.57 & 0.04 \\
\hline 19 & 9.5 & 69.78 & 0.04 & 39 & 19.5 & 143.24 & 0.04 \\
\hline 20 & 10 & 73.46 & 0.04 & 40 & 20 & 146.91 & 0.04 \\
\hline
\end{tabular}

As can be seen in the figure, the error can be less than $0.5 \mathrm{~m} / \mathrm{s}$.

Due to the influence of pressure on the wind speed calibration, and the temperature effect is relatively small, for the pressure calibration and temperature calibration, to be calibrated 1300, 1350, $1400,1450,1500 \mathrm{~Pa}$ three pressure conditions, temperature calibration $-120,-100,80$ three working conditions.

Calibration process is: the container is first pumped to a vacuum, pressurized to the lowest calibration pressure by nitrogen, adjusted to the highest temperature to be calibrated, calibrated at each speed, then repressurized to a second pressure, calibrated in sequence until the current pressure and velocity are calibrated, Heat sink to adjust the temperature to the next condition, until all conditions calibration completed.

\section{Conclusion}

This paper introduces the design method of low-pressure air velocity measurement system in detail, introduces the structure of the test system, selects the measuring sensor, considers the influence factors such as wire resistance and electromagnetic interference, and completes the design of the test flow to obtain the wind speed data under low pressure. Build a good foundation.

\section{References}

1. Johnson K. Simulation of Mars Surface conditions for Characterization of the Mars Rover Thermal Response[J].

2. Numata D, Anyoji M, Sugino Y, et al. Characteristics of Thermal Anemometers at Low-Pressure Condition in a Mars Wind Tunnel[C]// Aiaa Aerospace Sciences Meeting Including the New Horizons Forum and Aerospace Exposition. 2013.

3. Wilson C F. Measurement of wind on the surface of Mars[J]. University of Oxford, 2003.

4. Ransome T, Pesket S, Toplis G. Thermal Balance Testing of the Beagle2 Mars Lander[C]. International Symposium Environmental Testing for Space Programmes, 2001.

5. Quix H, Quest J, Brzek C. Hot-Wire Measurements in Cryogenic Environment[C]// Aiaa Aerospace Sciences Meeting Including the New Horizons Forum and Aerospace Exposition. 2013. 